Zbigniew Grande, Institute of Mathematics, Pedagogical University, ul. Chodkiewicza 30, 85-064 Bydgoszcz, Poland

e-mail: grande@wsp.bydgoszcz.pl

\title{
ON THE MEASURABILITY OF FUNCTIONS DEFINED ON THE PRODUCT OF TWO TOPOLOGICAL SPACES
}

\begin{abstract}
Some conditions implying the measurability of functions defined on the product of two topological spaces are investigated.
\end{abstract}

Let $\mathbb{R}$ denote the set of all reals and let $\left(X, \mathcal{T}_{X}\right)$ and $\left(Y, \mathcal{T}_{Y}\right)$ be topological spaces. Moreover, let $\mu_{1}$ and $\mu_{2}$ respectively, be $\sigma$-finite measures defined on some $\sigma$-fields $\mathcal{M}_{1} \supset \mathcal{T}_{X}$ and $\mathcal{M}_{2} \supset \mathcal{T}_{Y}$. Assume that

(1) for every set $A \in \mathcal{M}_{1}$ with $\mu_{1}(A)>0$ there is a set $B \in \mathcal{T}_{X}$ such that $B \subset A$ and $\mu_{1}(B)>0$;

(2) $\mu_{1}(A)>0$ for all nonempty sets $A \in \mathcal{T}_{X}$.

A function $f: X \rightarrow \mathbb{R}$ is called $\mathcal{T}_{X}$-quasicontinuous $\left(\mathcal{T}_{X}\right.$-cliquish) at a point $x \in X$ ([5] if for every positive real $\eta$ and for every set $U \in \mathcal{T}_{X}$ containing $x$ there is a nonempty set $V \in \mathcal{T}_{X}$ such that $V \subset U$ and $|f(v)-f(x)|<\eta$ for all points $v \in V\left(\operatorname{osc}_{V} f<\eta\right.$, where $\operatorname{osc}_{V} f$ denotes the diameter of the set $f(V))$.

In the proofs we will use the following Davies lemma ([2, 3]):

Lemma 1. Suppose that the measure $\mu_{1}$ is complete and a function $f: X \rightarrow \mathbb{R}$ is such that for every positive real $\eta$ and for every set $A \in \mathcal{M}_{1}$ with $\mu_{1}(A)>0$ there is a set $B \in \mathcal{M}_{1}$ such that $B \subset A, \mu_{1}(B)>0$ and $\operatorname{osc}_{B} f<\eta$. Then the function $f$ is $\mu_{1}$-measurable.

Remark 2. If a function $f: X \rightarrow \mathbb{R}$ is measurable with respect to $\mu_{1}$, then it is $\mathcal{T}_{X}$-cliquish at every point $x \in X$;

\footnotetext{
Key Words: continuity, quasicontinuity, cliquishness, measurability, density topology, product measure.

Mathematical Reviews subject classification: 28A35, 28A10, 54C08, 54C30

Received by the editors June 16, 1997

* Supported by Bydgoszcz Pedagogical University grant 1997
} 
If the measure $\mu_{1}$ is complete, then every function $f: X \rightarrow \mathbb{R}$ is $\mathcal{T}_{X}$-cliquish at each point is $\mu_{1}$-measurable.

Proof. Assume that the function $f$ is $\mu_{1}$-measurable and fix a positive real $\eta$, a point $x \in X$ and a set $U \in \mathcal{T}_{X}$ containing $x$. Since the function $f$ is $\mu_{1}$ measurable and $\mu_{1}(U)>0$, there is an open interval $I$ of the length $d(I)<\eta$ such that $\mu_{1}\left(f^{-1}(I) \cap U\right)>0$. By (1) there is a nonempty set $V \in \mathcal{T}_{X}$ such that $V \subset U \cap f^{-1}(I)$. Since $d(I)<\eta$, we obtain $\operatorname{osc}_{V} f<\eta$.

Now, we suppose that the function $f$ is $\mathcal{T}_{X}$-cliquish at every point. Fix a positive real $\eta$ and a set $A \in \mathcal{M}_{1}$ with $\mu_{1}(A)>0$. By (1) there is a nonempty set $U \in \mathcal{T}_{X}$ such that $U \subset A$. Fix a point $x \in U$. Since the function $f$ is $\mathcal{T}_{X}$-cliquish at $x$, there is a nonempty set $V \in \mathcal{T}_{X}$ such that $V \subset U$ and $\operatorname{osc}_{V} f<\eta$. Since $V \in \mathcal{M}_{1}$ and $\mu_{1}(V)>0$, we obtain by Davies's Lemma that the function $f$ is measurable with respect to $\mu_{1}$.

Remark 3. If a function $f: X \rightarrow \mathbb{R}$ is $\mu_{1}$-measurable and if $C(f)$ denotes the set of all $\mathcal{T}_{X}$-continuity points of $f$, then $\mu_{1}(X \backslash C(f))=0$.

Proof. Suppose, to the contrary, that $\mu_{1}(X \backslash C(f))>0$. For a point $x \in X$ let

$$
\operatorname{osc} f(x)=\inf \left\{d(f(A)) ; x \in A \in \mathcal{T}_{X}\right\},
$$

where $d(f(A))$ denotes the diameter of the set $f(A)$. Evidently,

$$
X \backslash C(f)=\bigcup_{n=1}^{\infty}\{x ; \text { osc } f(x) \geq 1 / n\} .
$$

So, there is a positive integer $n$ such that $A_{n}=\{x$; osc $f(x) \geq 1 / n\}$ is not of measure $\mu_{1}$ zero. Since the set $A_{n}$ is $\mathcal{T}_{X}$-closed, we have $A_{n} \in \mathcal{M}_{1}$ and $\mu_{1}\left(A_{n}\right)>0$. By (1) there is a nonempty set $U \in \mathcal{T}_{X}$ such that $U \subset A_{n}$. Fix a point $x \in U$. Since the function $f$ is $\mathcal{T}_{X}$-cliquish at $x$, there is a nonempty set $V \in \mathcal{T}_{X}$ such that

$$
\left(V \subset U \subset A_{n}\right) \wedge\left(\operatorname{osc}_{V} f<1 / n\right) .
$$

So, we obtain a contrary with the inequality osc $f(v) \geq 1 / n$ for $v \in V$.

Now, we will consider some functions of two variables.

For this, let $\mathcal{M}=\mathcal{M}_{1} \times \mathcal{M}_{2}$ and let $\mu$ be the completion of the product measure $\mu_{1} \times \mu_{2}$. Assume also that:

(3) For every set $A \in \mathcal{M}$ with $\mu(A)>0$ there is a set $B \in \mathcal{M}$ such that $(B \subset A) \wedge(\mu(B)>0)$, all sections

$$
B_{x}=\{y \in Y ;(x, y) \in B\} \in \mathcal{T}_{Y} ; x \in X,
$$


and all sections

$$
B^{y}=\{x \in X ;(x, y) \in B\} \in \mathcal{T}_{X} ; y \in Y .
$$

Let $\mathcal{A} \subset \mathcal{M}_{2}$ be a family of subsets of $Y$ of positive measure $\mu_{2}$, let $y \in Y$ be a point and let $f: Y \rightarrow \mathbb{R}$ be a function. We will write:

$f \in \mathcal{B}(\mathcal{A})$ if and only if for every positive real $\eta$ and for every set $U \in \mathcal{T}_{Y}$ there is a set $A \in \mathcal{A}$ such that

$$
\left(\mu_{2}(A \cap U)>0\right) \wedge\left(\operatorname{osc}_{A \cap U} f<\eta\right)
$$

$f \in \mathcal{Q}_{s}(y, \mathcal{A})$ if and only if for every positive real $\eta$ and for every set $U \in \mathcal{T}_{Y}$ containing $y$ there is a nonempty set $V \in \mathcal{A}$ such that

$$
\left(\mu_{2}(V \cap U)>0\right) \wedge(|f(t)-f(y)|<\eta)
$$

for all points $t \in V \cap U$.

Theorem 4. Let $f: X \times Y \rightarrow \mathbb{R}$ be a function such that all sections $f^{y}(x)=$ $f(x, y), x \in X$ and $y \in Y$, are $\mathcal{T}_{X}$-quasicontinuous at every point $x \in X$. If there is a countable family $\mathcal{A} \subset \mathcal{M}_{2}$ of subsets of $Y$ of positive measure $\mu_{2}$ such that for every point $x \in X$ the section $f_{x}(y)=f(x, y), y \in Y$, belongs to the family $\mathcal{B}(\mathcal{A})$, then the function $f$ is measurable with respect to the measure $\mu$.

Proof. We will prove that the function $f$ satisfies the hypothesis of Davies Lemma. Fix a positive real $\eta$ and a set $A \in \mathcal{M}$ such that $\mu(A)>0$. By (3) there is a set $B \in \mathcal{M}$ such that $(B \subset A) \wedge(\mu(B)>0)$ and all sections

$$
B_{x} \in \mathcal{T}_{Y}, x \in X \text { and } B^{y} \in \mathcal{T}_{X}, y \in Y .
$$

Enumerate all sets of the family $\mathcal{A}$ in a sequence (finite or not)

$$
A_{1}, \ldots, A_{n}, \ldots
$$

By our hypothesis for every point $(x, y) \in B$ there is a set $A(x, y) \in \mathcal{A}$ such that

$$
\operatorname{osc}_{A(x, y) \cap B_{x}} f_{x}<\eta / 8 \text { and } \mu_{2}\left(A(x, y) \cap B_{x}\right)>0 .
$$

Since $\mu(B)>0$, there is a positive integer $n$ such that the set

$$
D=\left\{(x, y) \in B ; A(x, y)=A_{n}\right\}
$$


is not of measure $\mu$ zero. Let

$$
\operatorname{Pr}_{X}(D)=\left\{x \in X ; \exists_{y}(x, y) \in D\right\}
$$

and let $E \subset X$ be a $\mu_{1}$-measurable covering of the set $\operatorname{Pr}_{X}(D)$, i.e. the set $E \in \mathcal{M}_{1}, \operatorname{Pr}_{X}(D) \subset E$ and every $\mu_{1}$-measurable set $S \subset E \backslash \operatorname{Pr}_{X}(D)$ is such that $\mu_{1}(S)=0$. Evidently, $\mu_{1}(E)>0$. By (1) there is a nonempty set $U$ such that $\left(U \in \mathcal{T}_{X}\right) \wedge(U \subset E)$. Fix a point $(x, y) \in\left(U \times A_{n}\right) \cap B$. Since $x \in U \cap B^{y}$ and the section $f^{y}$ is $\mathcal{T}_{X}$-quasicontinuous at $x$, there is a nonempty set $V \subset U \cap B^{y}$ such that

$$
\left(V \in \mathcal{T}_{X}\right) \wedge\left(\operatorname{osc}_{V} f^{y}<\eta / 8\right) .
$$

If points

$$
\left(u_{1}, v_{1}\right),\left(u_{2}, v_{2}\right) \in\left(\left(V \cap \operatorname{Pr}_{X}(D)\right) \times A_{n}\right) \cap B,
$$

then

$$
\begin{gathered}
\left|f\left(u_{1}, v_{1}\right)-f\left(u_{2}, v_{2}\right)\right| \leq\left|f\left(u_{1}, v_{1}\right)-f\left(u_{1}, y\right)\right|+\left|f\left(u_{1}, y\right)-f\left(u_{2}, y\right)\right| \\
+\left|f\left(u_{2}, y\right)-f\left(u_{2}, v_{2}\right)\right|<\eta / 8+\eta / 8+\eta / 8=3 \eta / 8
\end{gathered}
$$

So, there is a closed interval $I$ of the length $d(I) \leq 3 \eta / 8$ such that

$$
f\left(\left(\left(V \cap \operatorname{Pr}_{X}(D)\right) \times A_{n}\right) \cap B\right) \subset I .
$$

Let $J$ be the closed interval of the length $3 \eta / 4$ having the same center as $I$. Assume, to the contrary, that there is a point $(u, v) \in\left(V \times A_{n}\right) \cap B$ such that $f(u, v) \in \mathbb{R} \backslash J$. Since $u \in V \cap B^{v} \in \mathcal{T}_{X}$, by the $\mathcal{T}_{X}$-quasicontinuity of the section $f^{v}$ at the point $u$ we obtain the existence of a nonempty set $W \in \mathcal{T}_{X}$ such that

$$
W \subset V \cap B^{v} \wedge \forall_{w \in W} f(w, v) \in \mathbb{R} \backslash J .
$$

But

$$
\left(\mu_{1}(W)>0\right) \wedge(W \subset U \subset E),
$$

so there is a point

$$
s \in W \cap \operatorname{Pr}_{X}(D) \subset V \cap \operatorname{Pr}_{X}(D) .
$$

Observe that

$$
\left(v \in A_{n}\right) \wedge\left(s \in W \subset B^{v}\right) \wedge((s, v) \in B) .
$$

Since $s \in \operatorname{Pr}_{X}(D)$, we have $f(s, v) \in I$. So, we obtain a contradiction to $f(s, v) \in \mathbb{R} \backslash J$. 
By Fubini's theorem the $\mu$-measurable set $P=\left(V \times A_{n}\right) \cap B$ is of positive measure $\mu$. So,

$$
P \subset A \wedge \mu(P)>0 \text { and } \operatorname{osc}_{P} f \leq d(J)<\eta
$$

and, by Davies's Lemma, the function $f$ is $\mu$-measurable.

Remark 5. Observe that in Theorem 1, it is suffices to assume only that the sections $f_{x}$ belong to the family $\mathcal{B}(\mathcal{A})$ with the exception of a set of measure $\mu_{1}$ zero.

Remark 6. If $X=Y=\mathbb{R}, \mu_{1}=\mu_{2}$ is Lebesgue measure in $X=Y$ and $\mathcal{T}$ is the density topology (see [1]), then the conditions (1), (2), (3) are satisfied. There is a nonmeasurable (in the Lebesgue sense) set $A \subset \mathbb{R}^{2}$ such that all sections $A_{x}, A^{y}, x, y \in \mathbb{R}$, are empty or contain only one point (see [6]). Let $\mathcal{A}$ be the family of all open intervals with rational endpoints. If $f(x, y)=1$ for $(x, y) \in A$ and 0 otherwise on $\mathbb{R}^{2}$, then the function $f$ is nonmeasurable and the sections $f_{x}$ and $f^{y}, x, y \in \mathbb{R}$, are measurable and belong to $\mathcal{B}(\mathcal{A})$.

Theorem 7. Let $f: X \times Y \rightarrow \mathbb{R}$ be a function such that the sections $f^{y}$, $y \in \mathbb{R}$, are $\mu_{1}$-measurable. Suppose that there is a countable family $\mathcal{A} \subset \mathcal{M}_{2}$ of sets of positive measure $\mu_{2}$ such that for every positive real $\eta$ and for each nonempty set $U \in \mathcal{M}_{2}$ with $\mu_{2}(U)>0$ there is a set $A \in \mathcal{A}$ such that $A \subset U$ and for each point $x \in \mathbb{R}$ the relation $\operatorname{osc}_{A} f_{x}<\eta$ holds. Then the function $f$ is $\mu$-measurable.

Proof. Let $B \in \mathcal{M}$ be a set of positive measure $\mu$ and let $\eta$ be a positive real. There is a $\mu$-measurable set $D \subset B$ such that $\mu(D)>0$ and all sections $D_{x} \in \mathcal{T}_{Y}, x \in X$, and $D^{y} \in \mathcal{T}_{X}, y \in Y$. By Fubini's theorem and our hypothesis there are an interval $I$ and a set $A \in \mathcal{A}$ such that the set

$$
E=\left\{u \in D^{y} ; A \subset D_{u} \wedge f_{u}(A) \subset I\right\}
$$

is not of measure $\mu_{1}$ zero and $d(I)<\eta / 8$.

Let $F \subset A$ be a nonempty set belonging to $\mathcal{A}$ such that for each point $x \in X$ the inequality $\operatorname{osc}_{F} f_{x}<\eta / 8$ is true. If $G \supset E$ is $\mu_{1}$-measurable covering of the set $E$, then there is a nonempty set $H \subset G$ belonging to $\mathcal{T}_{X}$. Let $K=(H \times F) \cap D$ and let $J$ be a closed interval having the same center as $I$ and such that $d(J)=3 \eta / 4$. The set $K$ is $\mu$-measurable and $\mu(K)>0$. Observe that if for a point $x \in \mathbb{R}$ there is a point $t \in F$ such that $f(x, t) \in \mathbb{R} \backslash J$, then $f_{x}(F) \subset \mathbb{R} \backslash I$. Since the sections $f^{v}$ are $\mu_{1}$-measurable, the set

$$
S=\left\{u \in H: \exists_{t \in F} f(u, t) \in \mathbb{R} \backslash J\right\}
$$


is of measure $\mu_{1}$ zero. Consequently, $f(K \backslash(S \times F)) \subset J$. Since

$$
(K \backslash(S \times F) \subset B) \wedge(\mu(K \backslash(S \times F))>0),
$$

by Davies's Lemma, the function $f$ is $\mu$-measurable.

Theorem 8. Let $f: X \times Y \rightarrow \mathbb{R}$ be a function such that all sections $f^{y}$, $y \in Y$, are $\mu_{1}$-measurable. If there is a countable family $\mathcal{A} \subset \mathcal{M}_{2}$ of sets of positive measure $\mu_{2}$ such that for every point $x \in X$ and for every point $y \in Y$ the relation $f_{x} \in \mathcal{Q}_{s}(y, \mathcal{A})$ is true, then the function $f$ is $\mu$-measurable.

Proof. Fix a positive real $\eta$ and a set $A \in \mathcal{M}$ with $\mu(A)>0$. By (3) there is a $\mu$-measurable set $B \subset A$ such that $\mu(B)>0$ and

$$
\forall_{(x, y) \in B}\left[\left(B_{x} \in \mathcal{T}_{Y}\right) \wedge\left(B^{y} \in \mathcal{T}_{X}\right)\right] .
$$

Fix a point $(x, y) \in B$. By Remark 1 the section $f^{y}$ is $\mathcal{T}_{X}$-cliquish at the point $x$. From the $\mathcal{T}_{X}$-cliquishness of the section $f^{y}$ at $x$ it follows that there is a nonempty set $D$ such that

$$
D \subset B^{y} \wedge D \in \mathcal{T}_{X} \wedge \operatorname{osc}_{D} f^{y}<\eta / 20 .
$$

By our hypothesis for every point $u \in D$ there is a set $A(u) \in \mathcal{A}$ such that

$$
\begin{aligned}
& \mu_{2}\left(A(u) \cap B_{u}\right)>0 ; \\
& |f(u, t)-f(u, y)|<\eta / 20 \text { for every point } t \in A(u) \cap B_{u} .
\end{aligned}
$$

Since the family $\mathcal{A}$ is countable and $\mu_{1}(D)>0$, there is a set $E \in \mathcal{A}$ such that the set $F=\{u \in D ; A(u)=E\}$ is not of measure $\mu_{1}$ zero. Let $G$ be $\mu_{1}$-measurable covering of the set $F$ and let $H$ be a nonempty set such that $H \subset G \cap D \wedge H \in \mathcal{T}_{X}$. If points $\left(u_{1}, v_{1}\right),\left(u_{2}, v_{2}\right)$ belong to the set $(F \times E) \cap B$, then

$$
\begin{gathered}
\left|f\left(u_{1}, v_{1}\right)-f\left(u_{2}, v_{2}\right)\right| \leq\left|f\left(u_{1}, v_{1}\right)-f\left(u_{1}, y\right)\right|+\left|f\left(u_{1}, y\right)-f\left(u_{2}, y\right)\right| \\
+\left|f\left(u_{2}, y\right)-f\left(u_{2}, v_{2}\right)\right|<\eta / 20+\eta / 20+\eta / 20=3 \eta / 20 .
\end{gathered}
$$

So, there is a closed interval $I$ such that

$$
d(I) \leq 3 \eta / 20 \wedge f((F \times E) \cap B) \subset I .
$$

Let $J$ be the closed interval having the same center as $I$ and such that $d(J)=$ $3 \eta / 4$. Put $K=(H \times E) \cap B$. The set $K \in \mathcal{M}$ and by Fubini's theorem $\mu(K)>0$. Let $P \subset K$ be a $\mu$-measurable set such that $\mu(P)>0$ and

$$
\forall_{(u, v) \in P}\left[\left(P_{u} \in \mathcal{T}_{Y}\right) \wedge\left(P^{v} \in \mathcal{T}_{X}\right)\right] .
$$


We will prove that $\mu\left(P \backslash f^{-1}(J)\right)=0$. Assume, to the contrary, that the set $L=P \backslash f^{-1}(J)$ is not of measure $\mu$ zero. Then for every point $(u, v) \in L$ there is a set $B(u, v) \in \mathcal{A}$ such that

$$
\begin{aligned}
& \mu_{2}\left(B(u, v) \cap P_{u}\right)>0 ; \\
& f(u, w) \in \mathbb{R} \backslash J \text { for every point } w \in B(u, v) \cap P_{u} .
\end{aligned}
$$

Since the family $\mathcal{A}$ is countable, there is a set $N \in \mathcal{A}$ such that the set

$$
M=\{(u, v) \in L ; B(u, v)=N\}
$$

is not of measure $\mu$ zero. Let $M_{1}$ be a $\mu_{1}$-measurable covering of the projection $\operatorname{Pr}_{X}(M)$ and let $Q \in \mathcal{T}_{X}$ be a nonempty set contained in the set $M_{1} \cap H$. Evidently,

$$
\begin{aligned}
& S=(Q \times N) \cap P \in \mathcal{M} ; \\
& \mu(S)>0 ; \\
& f((M \times N) \cap P) \subset \mathbb{R} \backslash J .
\end{aligned}
$$

Fix a point $(u, v) \in S$. Since the section $f^{v}$ is $\mathcal{T}_{X}$-cliquish at the point $u$, there is a nonempty set $U \in \mathcal{T}_{X}$ such that

$$
\left(U \subset Q \cap P^{v}\right) \wedge\left(\operatorname{osc}_{U} f^{v}<\eta / 20\right) .
$$

There are points $(s, v),(t, v)$ belonging to $S$ with $s \in U \cap D$ and $t \in U \cap$ $\operatorname{Pr}_{X}(M)$. Then $(f(s, v) \in I) \wedge(f(t, v) \in \mathbb{R} \backslash J)$. Consequently, we obtain

$$
|f(s, v)-f(t, v)| \geq 3 \eta / 8-3 \eta / 40>\eta / 5,
$$

and $\operatorname{osc}_{U} f^{v}>\eta / 20$. This contradiction shows that $\mu\left(P \backslash f^{-1}(J)\right)=0$. The set $P \cap f^{-1}(J) \subset A$ is $\mu$-measurable, $\mu\left(P \cap f^{-1}(J)\right)>0$ and

$$
\operatorname{osc}_{\left(P \cap f^{-1}(J)\right)} f \leq d(J)<\eta \text {. }
$$

Hence, by Davies's Lemma, the function $f$ is $\mu$-measurable.

Particular cases of properties $\mathcal{B}(\mathcal{A})$ and $\mathcal{Q}_{s}(\mathcal{A})$ are investigated in $[3,4]$.

\section{References}

[1] A. M. Bruckner, Differentiation of real functions, Lecture Notes in Math. 659, Springer-Verlag, Berlin 1978. 
[2] R. O. Davies, Separate approximate continuity implies measurability, Math. Proc. Camb. Phil. Soc. 73 (1973), 461-465.

[3] Z. Grande, La mesurabilité des fonctions de deux variables et de la superposition $F(x, f(x))$, Dissert. Math.159 (1978), 1-50.

[4] Z. Grande, On strong quasi-continuity of functions of two variables., Real Analysis Exch. 21 No.1 (1995-96), 236-243.

[5] T. Neubrunn, Quasi-continuity, Real Analysis Exch. 14 No.2 (1988-89), 259-306.

[6] W. Sierpiński, Sur un problème concernant les ensembles mesurables superficiellement, Fund. Math. 1 (1920), 112-115. 